\title{
Emergência de Haematobia irritans em fezes bovinas no município de Seropédica, Rio de Janeiro ${ }^{1}$
}

\author{
Douglas Marques de Macedo² ${ }^{2}$ Luciana Gatto Brito ${ }^{2}$ e Gonzalo Efrain Moya Borja ${ }^{3}$
}

\begin{abstract}
Macedo D.M., Brito L.G. \& Moya Borja G.E. 2001. [Emergence of Haematobia irritans in cattle dung pats in Seropédica county, Rio de Janeiro] Emergência de Haematobia irritans em fezes bovinas no município de Seropédica, Rio de Janeiro. Pesquisa Veterinária Brasileira 21(2):77-80. Depto Parasitologia Animal, Univ. Fed. Rural do Rio de Janeiro, Seropédica, RJ 23851-970, Brazil.

This study was carried out in order to investigate the emergence of adult flies of Haematobia irritans in cattle dung maintained in the field and in the laboratory, as well as other flies associated with dung pats. Two groups of cattle dung were used, one in the field and another in the laboratory, each group consisting of five fresh dung pats of medium size (about $30 \mathrm{~cm}$ in diameter), opaque appearance, presence of a thin external crust and firm consistency. The dung pats were covered with emergence cages in pyramidal format, presenting a hole in the superior part where a removable flask was coupled, and the substitution of the dung was done every 2 weeks. A total of 355 specimens of $H$. irritans was collected, 151 of which were obtained in the field and 204 specimens in the laboratory. These differences probably were due to smaller temperature variations in the laboratory than in the field, besides the action of arthropodal predators, competitors and parasites of horn fly pupae that probably interfered in $H$. irritans emergence in cattle dung under field conditions. A higher number of females were observed in both conditions. Besides $H$. irritans, there were collected other flies associated with bovine manure belonging to the following families: Aulacigastridae, Muscidae, Psychodidae, Sarcophagidae, Sepsidae, Tachinidae and Ulidiidae. Sepsidae was the most abundant, with 5,224 specimens of the total of 8,928 flies collected, followed by Sarcophagidae with 2,235, Muscidae with 1,357, Aulacigastridae with 54 , Psychodidae with 46 , Ulidiidae with 6 and Tachinidae with 5 specimens.
\end{abstract}

INDEX TERMS: Haematobia irritans, emergency, bovine manure.

RESUMO.- O presente trabalho teve como objetivo estudar a emergência de Haematobia irritans em fezes de bovinos mantidas a campo e em laboratório, além de observar a presença de outros dípteros associados às massas fecais. Foram utilizados dois grupos de fezes bovinas, um à campo e outro em laboratório, sendo cada grupo formado por cinco bolos fecais frescos de tamanho mediano (cerca de $30 \mathrm{~cm}$ de diâmetro), aparência opaca, cor esverdeada, presença de fina crosta externa e consistência firme. As fezes foram cobertas

\footnotetext{
${ }^{1}$ Aceito para publicação em 7 de maio de 2001.

Trabalho realizado sob os auspícios do CNPq.

${ }^{2}$ Doutorando CPMV-PV, Universidade Federal Rural do Rio de Janeiro (UFRRJ), Seropédica, RJ 23851-970; e-mail: macedo@ufrrj.br

${ }^{3}$ Depto Parasitologia Animal, UFRRJ; e-mail:gemoya@ufrrj.br
}

com gaiolas de emergência de formato piramidal, apresentando na parte superior um orifício onde foi acoplado um frasco removível; a substituição das fezes foi realizada quinzenalmente. Foram coletados um total de 355 espécimens de $H$. irritans, sendo 151 provenientes das gaiolas mantidas no campo e 204 em laboratório. Estas diferenças deveram-se provavelmente à menores oscilações de temperatura verificadas no laboratório do que àquelas ocorridas no campo, além da ação de artrópodes predadores, competidores e parasitóides que, provavelmente, interferiram na emergência de $H$. irritans nas fezes bovinas mantidas no campo. Foi observado maior número de fêmeas em comparação com machos em ambas as condições investigadas. Além de $H$. irritans, obteve-se outros dípteros associados às fezes bovinas, pertencentes as seguintes famílias: Aulacigastridae, Muscidae, Psychodidae, Sarcophagidae, Sepsidae, Tachinidae e Ulidiidae. Sepsidae foi a mais abundante, com 5.224 exem- 
plares do total de 8.928 dípteros obtidos, seguida por Sarcophagidae com 2.235 espécimens coletados, Muscidae com 1.357, Aulacigastridae com 54, Psychodidae com 46, Ulidiidae com 6 e Tachinidae com 5 exemplares.

TERMOS DE INDEXAÇÃO: Haematobia irritans, emergência, fezes bovinas.

\section{INTRODUÇÃO}

Portchinsky (apud Hammer 1941), foi o primeiro autor a considerar fezes bovinas como uma unidade ecológica, por suportarem uma fauna de dípteros bastante diversificada, com espécies coprófagas, predadoras e parasitas de outras espécies. Estes dípteros são atraídos devido a disponibilidade de alimentos e pela presença de abrigos encontrados nas fezes bovinas.

A contribuição mais elaborada sobre esse assunto foi realizada por Mohr (1943), na Califórnia, Estados Unidos da América (EUA), quando ratificou as informações anteriores e verificou todo o complexo de dípteros, coleópteros e himenópteros existentes nas fezes analisadas. $\mathrm{O}$ autor identificou 36 espécies de 17 famílias de dípteros envolvidas com fezes frescas de bovino, concluindo que fatores físicos como odor, cor, forma e idade influenciaram na ocorrência e na frequiência das espécies. Comprovou, também, a atratividade deste substrato para espécies das famílias Sepsidae, Sphaeroceridae, Muscidae, Sarcophagidae, Stratiomyidae e Anthomyidae. Demonstrou, ainda, que os dípteros adultos foram abundantes na superfície do bolo fecal fresco; e observou que larvas e pupas eram encontradas em fezes mais envelhecidas, quando serviram de alimentos disponíveis para espécies de coleópteros e himenópteros. Concluiu, que os dípteros são atraídos em maior número e diversidade por fezes bovinas frescas, utilizando deste substrato em algum momento de seu ciclo de vida, ressaltando a ocorrência de espécies sinantrópicas.

Durante o verão de 1962-1963, Sanders \& Dobson (1966) em Lafayete, Indiana, EUA, coletaram 20 espécies de dípteros, 15 de coleópteros, três de himenópteros e dois de ácaros, que se desenvolveram em 98 amostras de fezes bovinas expostas ao meio ambiente por um período que variou de uma a 120 horas. Concluíram que somente os dípteros foram atraídos por fezes frescas; enquanto que, os outros grupos preferiram fezes envelhecidas. Também informaram que algumas espécies de dípteros utilizam as fezes frescas apenas como fonte alimentar, preferindo outros meios para o desenvolvimento larval, sendo as famílias Muscidae e Sarcophagidae as mais abundantes numericamente.

$\mathrm{O}$ bolo fecal bovino passa por vários processos físicos e biológicos que vão desde a diminuição de área e volume (desidratação), até sua total desintegração. As ações físicas devem-se à diminuição da umidade pela evaporação; à fragmentação, devido às variações de temperatura e umidade; ao crescimento de uma nova vegetação entre os fragmentos fecais e aos efeitos pluviométricos e eólicos. As ações biológicas são promovidas por artrópodes, pássaros, mamíferos e microorganismos (Merrit \& Anderson 1977, Anderson et al. 1984,
Hanski 1987, Barth et al. 1994). Os insetos cumprem papel importante no processo de incorporação de nutrientes ao solo, uma vez que as interações entre eles e deles com o bolo fecal agilizam a incorporação das fezes no solo (Fincher 1986, Miranda et al. 2000).

As interações existentes entre os artrópodes demonstram a importância da realização deste experimento, que teve como principal objetivo verificar a emergência de $H$. irritans em fezes bovinas mantidas a campo e em laboratório, bem como, de outros dípteros associados ao bolo fecal.

\section{MATERIAL E MÉTODOS}

Durante o período de agosto de 1995 a julho de 1996, quinzenalmente, foram coletados aleatoriamente na Estação de Pesquisas Parasitológicas William Otton Neitz, do Departamento de Parasitologia Animal do Instituto de Veterinária da Universidade Federal Rural do Rio de Janeiro, cinco bolos fecais frescos de bovinos que apresentavam as seguintes características: tamanho mediano (com aproximadamente $30 \mathrm{~cm}$ de diâmetro), aparência opaca, cor esverdeada, fina crosta externa, consistência firme, que estavam intactos e distribuídos aleatoriamente por uma área de $40.000 \mathrm{~m}^{2}$ contendo 12 bovinos adultos durante todo o período experimental.

Cada amostra foi recolhida com uma pá de borda retilínea, com a qual procurou-se colher todo o resquício fecal e alguma quantidade do solo de contato, sendo as fezes acondicionadas em caixa plástica (50 $\mathrm{cm}$ de comprimento, $40 \mathrm{~cm}$ de largura e $12 \mathrm{~cm}$ de altura). No laboratório, sob condições ambientais de temperatura e de umidade relativa do ar, cada bolo fecal foi colocado sob gaiola de madeira (45cm de comprimento, $45 \mathrm{~cm}$ de largura e $45 \mathrm{~cm}$ de altura) construída sob os critérios preconizados por Kunz et al. (1970), revestida com tela de náilon e de formato piramidal, apresentando um orifício na parte superior onde fora conectado um frasco removível cujo interior continha um funil de polietileno que impedia o refluxo dos insetos que alcançassem o frasco. Foi colocado barro úmido e compactado entre a parte inferior da gaiola e o solo, para evitar o acesso de possíveis predadores ao interior das gaiolas.

Após a emergência, os insetos foram mortos com auxílio de éter e em seguida, transferidos para frascos de vidro $(10 \mathrm{~cm}$ de altura e $7 \mathrm{~cm}$ de diâmetro) devidamente rotulados, contendo álcool $70 \%$.

No experimento a campo, foram utilizadas cinco gaiolas, também construídas sob os critérios preconizados por Kunz et al. (1970). Estas gaiolas foram colocadas paralelamente e distantes um metro entre si em uma área de pastagem da estação experimental mantidas sob a influência direta das condições ambientais, sendo esta área isolada com arame farpado. As fezes foram trocadas quinzenalmente, sendo que após a emergência, utilizou-se a mesma metodologia para preservação e triagem dos insetos, como citado anteriormente.

Os dados meteorológicos, tais como temperatura, umidade relativa do ar e precipitação total, foram obtidos junto a Estação Ecologia Agrícola/PESAGRO, localizada no município de Seropédica (latitude sul $22^{\circ} 45^{\prime}$, longitude oeste $43^{\circ} 41^{\prime}$ ).

O delineamento estatístico utilizado para a comparação das médias de emergência de macho e fêmea, tanto a campo quanto em laboratório, foi o teste de Tukey-Kramer à nível de $5 \%$ de significância, associado ao teste de comparação múltipla de Bonferroni $(a=5 \%)$.

\section{RESULTADOS}

Houve diferença significativa entre o número de $H$. irritans obtidas de fezes mantidas no campo e àquelas mantidas em laboratório $(\mathrm{F}=4,325)$ (Fig. 1 e 2). Foram coletadas, em labo- 

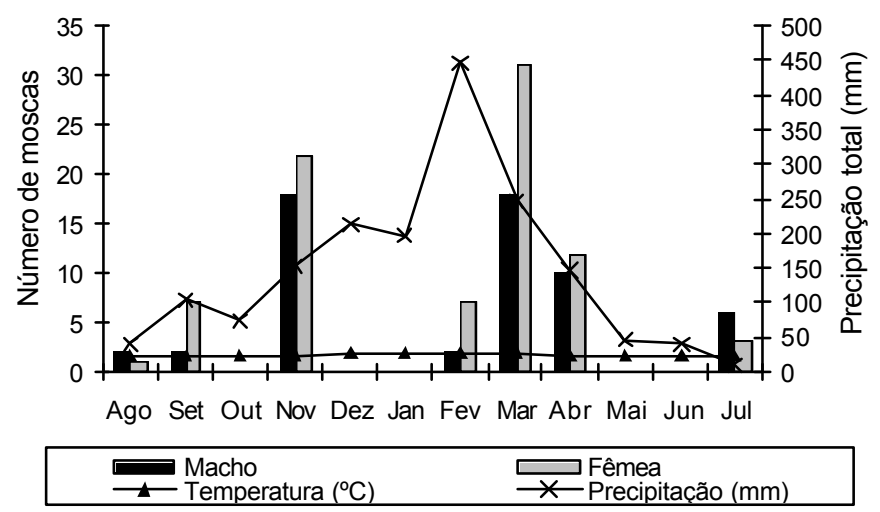

Fig. 1. Distribuição de adultos de Haematobia irritans provenientes de fezes bovinas acondicionadas em gaiolas no campo, no período de agosto de 1995 a julho de 1996, em Seropédica, RJ.
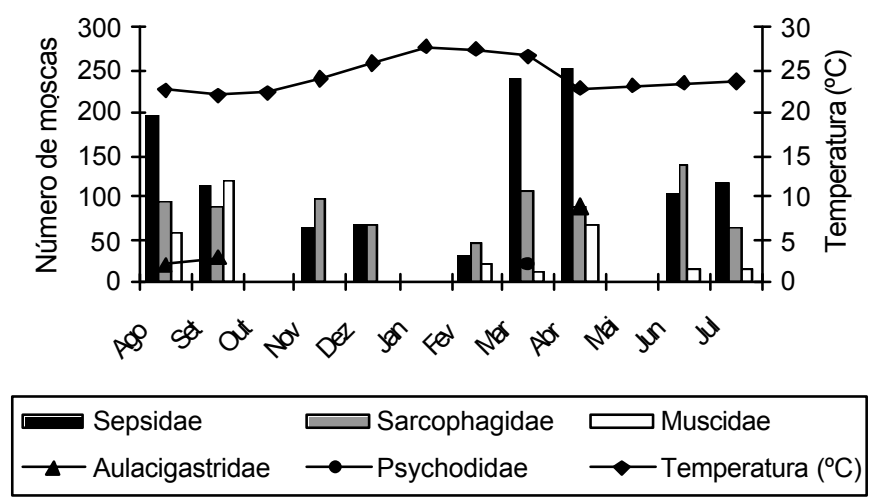

Fig. 3. Distribuição das duas famílias de dípteros provenientes de fezes bovinas acondicionadas em gaiolas no campo, no período de agosto de 1995 a julho de 1996, em Seropédica, RJ.

ratório, um total de 204 "moscas do chifre"; enquanto que, no campo foram obtidas 151. O teste de Bonferroni demonstrou uma maior emergência de machos nas gaiolas mantidas no campo, quando comparado a emergência de machos e fêmeas no laboratório.

No experimento realizado a campo, não foram obtidas $H$. irritans nos meses de outubro e dezembro de 1995, e janeiro, maio e junho de 1996 (Fig. 1) e nas outras famílias de dípteros estudados, não houve emergência nos meses de outubro de 1995, e janeiro e maio de 1996 (Fig. 3).

Durante o período experimental, foram obtidas nas fezes bovinas, além de $H$. irritans pertencente a família Muscidae, outras famílias de dípteros, a saber: Aulacigastridae, Muscidae, Psychodidae, Sarcophagidae, Sepsidae, Tachinidae e Ulidiidae (Fig. 3 e 4).

A família Sepsidae foi a mais abundante em número de indivíduos, com $5.224(58,51 \%)$ exemplares do total de 8.928 dípteros obtidos. Os Sarcophagidae aparecem a seguir com $2.235(25,03 \%)$ espécimens coletados, Muscidae com 1.357 $(15,20 \%)$, Aulacigastridae com $54(0,60 \%)$, Psychodidae com $46(0,51 \%)$, Ulidiidae com seis $(0,07 \%)$ e Tachinidae com cinco $(0,06 \%)$ exemplares.

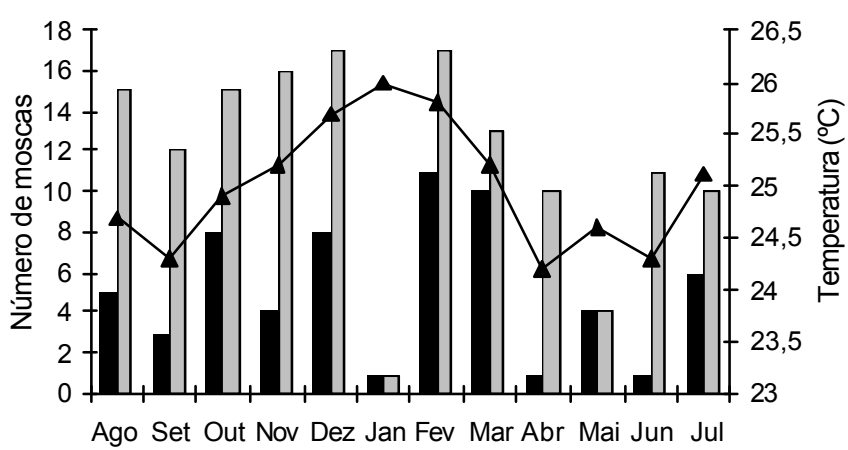

$\square$ Macho $\rightleftarrows$ Fêmea $\leadsto$ Temperatura $\left({ }^{\circ} \mathrm{C}\right)$

Fig. 2. Distribuição de adultos de Haematobia irritans provenientes de fezes bovinas acondicionadas em gaiolas no laboratório, no período de agosto de 1995 a julho de 1996, em Seropédica, RJ.

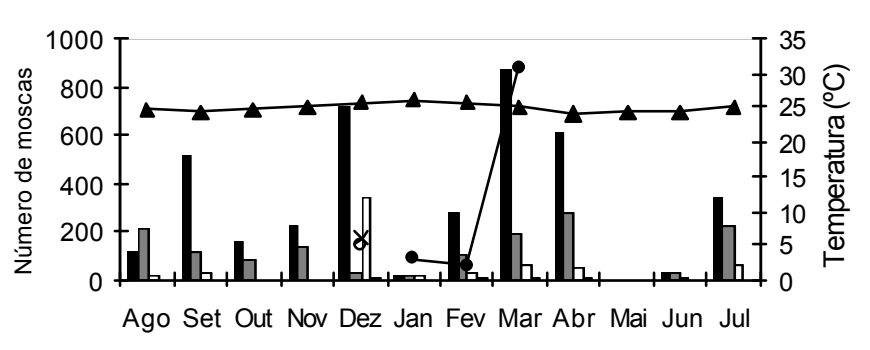

\begin{tabular}{|c|c|c|}
\hline $\begin{array}{l}\text { Sepsidae } \\
\text { Aulacigastridae } \\
\text { ○_ Tachinidae }\end{array}$ & $\begin{array}{l}\square \text { Sarcophagidae } \\
\longrightarrow \text { Psychodidae } \\
\simeq \text { Temperatura }\left({ }^{\circ} \mathrm{C}\right)\end{array}$ & $\begin{array}{l}\longleftarrow \text { Muscidae } \\
\star \text { Ulidiidae }\end{array}$ \\
\hline
\end{tabular}

Fig. 4. Distribuição das famílias de dípteros provenientes de fezes bovinas acondicionadas em gaiolas no laboratório, no período de agosto de 1995 a julho de 1996, em Seropédica, RJ.

\section{DISCUSSÃO}

A ocorrência de machos de $H$. irritans, em ambos os experimentos, foi menor em relação ao número de fêmeas, corroborando com os resultados obtidos por Linhares (1979), D'Almeida (1983), Lomônaco (1987), que trabalharam com levantamento de dípteros no campo, com emprego de diferentes substratos para atração.

As diferenças encontradas entre o número de moscas obtidas no campo e no laboratório, devem-se, provavelmente, à menores variações de temperatura no ambiente do laboratório do que no campo, além de uma maior umidade relativa do ar; enquanto que, no campo, a baixa emergência estaria associada à presença de artrópodes predadores, competidores e parasitóides que estariam interferindo na emergência e sobrevivência de H. irritans (Kunz et al. 1970, Kunz \& Cunningham 1977, Collares 1990).

Não foram verificadas emergências de $H$. irritans, no campo, nos meses de outubro, dezembro, janeiro, maio e junho. Também não houve emergência de outras famílias de dípteros nos meses de outubro, janeiro e maio, o que coincidiu com a ocorrência de intensas chuvas após a colocação das gaiolas, que não apenas reduziram drasticamente a população adul- 
ta da "mosca do chifre", como também diminuíram a população das fazes imaturas por destruírem parcialmente as massas fecais mais recentemente depositadas, nas quais as fases imaturas estariam presente.

Segundo Oliveira et al. (1993), os representantes da família Sepsidae, apesar de serem abundantes no bolo fecal bovino, apresentam pouca importância como competidores de H. irritans ou na degradação do bolo fecal. Estes autores observaram que as larvas dos sepsídeos contribuem no processo de aeração das fezes, devido às galerias que escavam, o que facilita a colonização por outros artrópodes e a diminuição da umidade das fezes.

D'Almeida (1983), em levantamento realizado utilizando diferentes substratos, observou a predominância de Calliphoridae com 42,3\%, seguido da família Muscidae com $26,8 \%$, Fannidae com $19,4 \%$ e Sarcophagidae com $11,4 \%$, sendo que no presente estudo com fezes bovinas, observou-se que os representantes da família Muscidae representavam a terceira família mais abundante.

\section{REFERÊNCIAS}

Anderson J.R., Merritt R.W. \& Loomis E.C. 1984. The insect-free cattle dung fouling of rangeland pastures. J. Econ. Entomol. 77:133-141.

Barth D., Karrer M., Heinze- Mustz E. M. \& Elster N. 1994. Colonization and degradation of cattle dung: aspects of sampling, fecal composition, and artificially formed pats. Environ. Entomol. 23(3):571-578.

Collares N.C.P. 1990. Alguns aspectos etológicos da Haematobia irritans em Roraima. Dissertação de Mestrado, Universidade Federal de Minas Gerais, Belo Horizonte, MG. 65 p.

D’Almeida J.M. 1983. Sinantropia em dípteros caliptratos na área metropolitana do Rio de Janeiro. Dissertação de Mestrado, Universidade Federal Rural do Rio de Janeiro, Itaguaí, RJ. 193 p.
Fincher G.T. 1986. Importation, colonization and release of dung-buryng scarabs, p. 69-76. In: Patterson R.S. \& Rutz D.A. (ed.) Biological Control of Muscoid Flies. Misc. Publ. Entomol. Soc. Am. 62:174.

Hammer O. 1941. Biological and ecological investigations on flies associated with pasturing cattle and their excrement. Vidensk. Medd. Dan. Naturhist. Foren. Kbhj. 105:1-257.

Hanski I. 1987. Nutritional ecology of dung- and carion-feeding insects, p. 839-841. In: Slaniki A. Jr \& Rodrigues J.G. (ed.) Nutritional Ecology of Insects, Mites, Spiders and related Invertebrates. John Wiley \& Sons, New York.

Kunz S.E., Blume R.R., Hogan B.F. \& Matter J.J. 1970. Biological and ecological investigations of horn flies in Central Texas: Influence of time of manure deposition on ovoposition. J. Econ. Entomol.63(3):920-923.

Kunz S.E. \& Cunningham J.R.. 1977. A population prediction equation with notes on the biology of the horn fly in Texas. Southwest. Entomol. 2(2):7987.

Linhares A.X. 1979. Sinantropia de dípteros muscóides de Campinas. Dissertação de Mestrado. Universidade de Campinas, Campinas, SP. 129p.

Lomônaco C. 1987. Ecologia comunitária da dipterofauna de muscóides da Restinga de Jacarepaguá, Rio de Janeiro, RJ. Dissertação de Mestrado, Universidade do Estado do Rio de Janeiro, Rio de Janeiro, RJ. 115p.

Merrit R.W. \& Anderson J.R. 1977. The effects of different pasture and rangeland ecosystems on the annual dynamics of insects in cattle droppings. Hilgardia 45(2):31-71.

Miranda C.H.B., Santos J.C. \& Bianchin I. 2000. The role ofDigitonthophagus gazella in pasture cleaning and production as result of burial cattle dung. Pasturas Tropicales 22(1):14-18.

Mohr C.O. 1943. Cattle dropping as ecological units. Monograf. 13:275-309.

Oliveira G.P., Mendes J. \& Dutra S.A.H. 1993. Abundância relativa da entomofauna simbovina na região de São Carlos, São Paulo. Ocorrência das principais espécies. Anais $45^{\mathrm{a}}$ Reunião Anual da SBPC, p. 946.

Sanders D.A. \& Dobson R.C. 1966. The insect complex associated with bovine manure in Indiana. Ann. Entomol. Soc. Am. 59:955-959. 